\title{
New Formulation for Semi-Empirical Correlations for Penetration Jets
}

\author{
R. R. Pacheco ${ }^{*}$, L. O. Freire' ${ }^{2}$, M. S. Rocha ${ }^{2}$, N. L. Scuro ${ }^{2}$, M. O. Menezes' ${ }^{2}$ D. A. Andrade ${ }^{2}$ \\ ${ }^{1}$ Centro Tecnológico da Marinha em São Paulo, São Paulo, Brazil \\ ${ }^{2}$ Instituto de Pesquisas Energéticas e Nucleares, São Paulo, Brazil \\ Email: ^rafaelrade@gmail.com, *delvonei@ipen.br
}

How to cite this paper: Pacheco, R.R., Freire, L.O., Rocha, M.S., Scuro, N.L., Menezes, M.O. and Andrade, D.A. (2019) New Formulation for Semi-Empirical Correlations for Penetration Jets. World Journal of Nuclear Science and Technology, 9, 96-111. https://doi.org/10.4236/wjnst.2019.92007

Received: January 22, 2019

Accepted: April 27, 2019

Published: April 30, 2019

Copyright (C) 2019 by author(s) and Scientific Research Publishing Inc. This work is licensed under the Creative Commons Attribution International License (CC BY 4.0).

http://creativecommons.org/licenses/by/4.0/

\begin{abstract}
Correlations for the extension of a water vapor jet injected in a liquid pool were historically proposed considering the mass flux $\left(\mathrm{kg} / \mathrm{m}^{2} / \mathrm{s}\right)$ as a constant. The results were satisfactory, however adjusting the values by linear regression. Although, it presents the following drawbacks: 1) the formulation is only valid for the specific range of data for what it was created; 2) it does not allow the analytical evaluation of the heat transfer coefficient from the extension equation. This paper proposes a new formulation for the calculation of the mass flux, in such a way to remove both of these drawbacks.
\end{abstract}

\section{Keywords}

Direct Contact Condensation, Vapor Jet, Steam Jet, Steam Mass Flux, Vapor Cavity, Reynolds Number, Jet Radius, Rate of Condensation, Non-Dimensional Jet Length, Liquid Pool, Dimensionless Transport Modulus, Condensation Driving Potential

\section{Introduction}

The phenomenon of Direct Contact Condensation (DCC) has been discussed in the literature since Kerney [1], due to its importance as a solution of engineering, where large values of heat transfer coefficient are needed. Through the DCC, several different sets of equipment can be designed, when, for example, condensation is in a small frame of time, or when reduced space is required.

DCC is the natural solution for applications where superheated vapor must be discharged in the atmosphere. Within this scope, DCC offers a safe alternative to reduce the heat and pressure up to the threshold where the discharge to the atmosphere is plausible, as it occurs in relief tanks.

For the nuclear industry, it emerges as a solution for containing the discharge 
of the high energetic water contained in a primary loop of a Pressure Water Reactor (PWR), and also, to direct it to the chemical and radiological plant treatment facilities.

For Boiling Water Reactors (BWR), DCC emerges as a solution to recollect the water vaporized within the reactor vessel. For this, BWR plants prescribe Suppression Pools in their projects, where the DCC phenomenon takes place.

The DCC phenomenon reached well-established economic importance and has become an object of research since it is related to the safety of nuclear power plants, where it occurs as a high energetic vapor injection in a tank containing liquid compressed water. The heat transfer coefficient is increased due to the turbulent character of the interface of the jet and the bath, and this is the key to the efficiency of thermal exchange [2].

The design of devices where the DCC may occur determined the interest in the research of the phenomenon. The research focused mainly on the determination of a model for the extension of the jet, and another model for the determination of the heat transfer coefficient.

Nevertheless, the highly complex physics of this phenomenon restrains the development of a full analytical model for the extension of the jet and for its heat transfer coefficient. Experimental data are supplied to fulfill the gaps in the analytical development and semi-empirical correlations have been proposed, observing the following restrictions: 1 ) these correlations are valid only for the specific range of experimental data; 2) the analytical evaluation of the heat transfer directly from the length correlation was not possible, considering the available formulation.

The present work proposes a new formulation for the flux of mass in the currently presented correlations in the literature, in such a way that their validity would extend to any set of data, and which would allow a direct deduction of the heat transfer coefficient from the adjusted correlation of the dimensionless extension. This proposition grounds itself in an analytical procedure considering the $1^{\text {st }}$ and the $2^{\text {nd }}$ law of the thermodynamics.

\section{Literature Review}

The first proposal to evaluate the extension of the jet, as in [1], is a development of the mass conservation, applied to a simple model, in which a superheated vapor jet produces a cavity full of vapor, discharged in an atmospheric water bath, as presented in Figure 1. In Equation (1), the vapor flow rate (“ $\dot{m}$ ”) along the injection axis " $x$ " is related to the jet radius " $r$ " and the rate of condensation " $R$ ".

$$
\frac{\mathrm{d} \dot{m}}{\mathrm{~d} x^{\prime}}=-2 \pi r^{\prime} R
$$

Figure 2 presents the basic configuration of the experimental set. A pressure chamber $\left(P_{C}\right)$ supplies superheated vapor. After the restriction by a valve, the vapor is injected in the water pool. The setting of the experiment allows a choked flow injection since it would reduce fluctuations in the jet. 


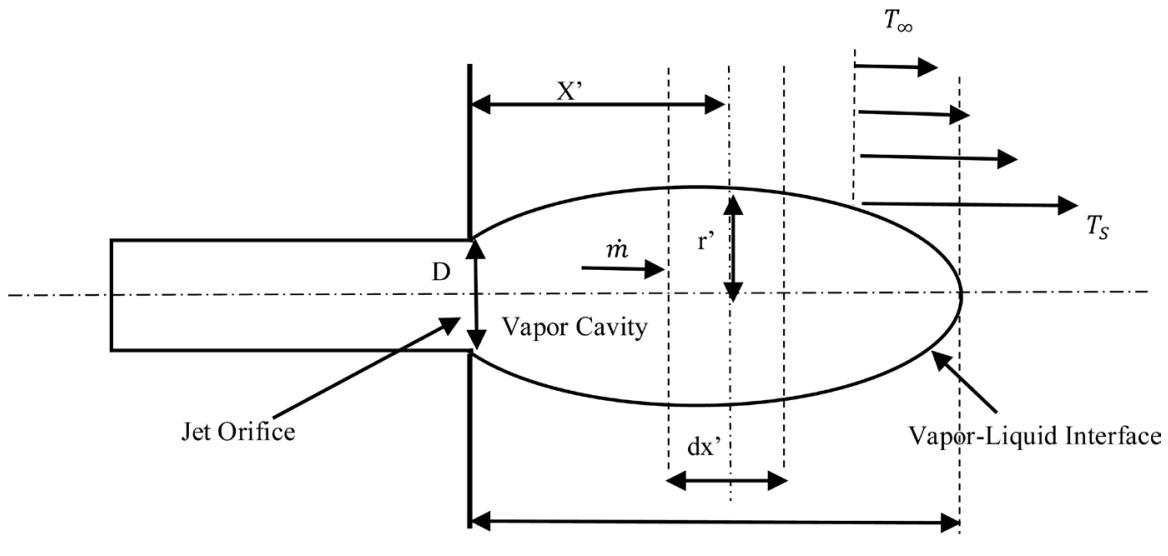

L

Figure 1. Dimensional variables [1].

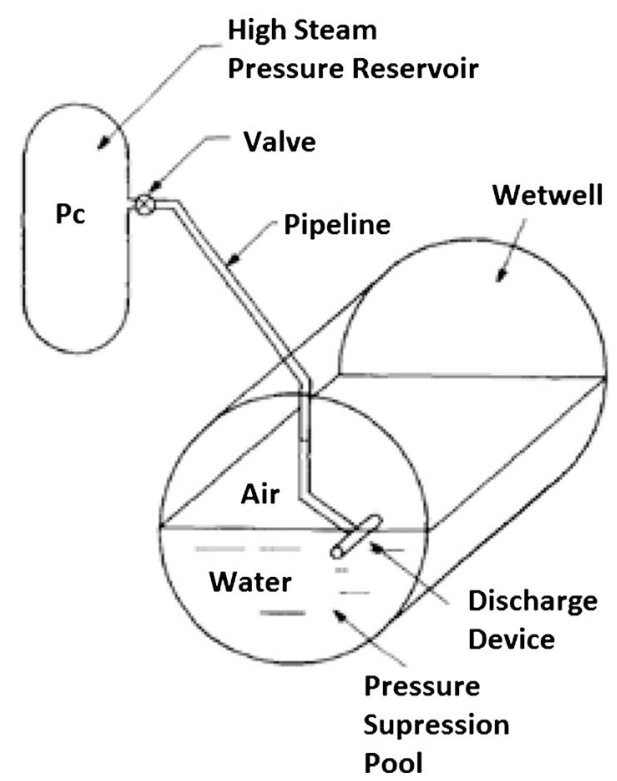

Figure 2. Illustration of the Experiment from (Sonin, 1981).

The dimensions $r^{\prime}$ and $\mathrm{d} x^{\prime}$ are depicted in Figure 1. According to this proposal, there is an outflow of water along the lateral vapor-liquid interface of the jet. This amount of water, crossing the jet interface, promptly freezes, assuming the properties of the surrounding water bath. This condensation is governed by $R$, which takes the following form:

$$
R=h \frac{\left(T_{S}-T_{\infty}\right)}{h_{g f}}
$$

The mass flow (Equation (3)) also takes part in this development. Initial conditions are applied in Equation (4).

$$
\begin{gathered}
\dot{m}=\pi r^{\prime 2} G \\
\dot{m}_{0}=\pi r_{0}^{\prime 2} G_{0}
\end{gathered}
$$

After some algebraic effort, Equation (1) and Equation (4) produce Equation 
(5):

$$
X=\int_{1}^{0}\left[-\left(\frac{G_{0}}{G}\right)^{1 / 2} \cdot B^{-1} \cdot S^{-1}\right] \mathrm{d} Y
$$

where $B$ is defined as the driving potential for condensation (Equation (6)), and $S$ as the dimensionless transport modulus, which is analogous to the Stanton Number (Equation (7)), although it presents some unorthodoxy, since the heat transfer coefficient $(h)$ and the specific heat $\left(C_{P}\right)$ are related to the liquid phase, and the mass flow rate $(G)$ is related to the vapor phase. $X$ is defined as the dimensionless jet length, Equation (8). For sake of clarity, $Y$ replaces the term presented Equation (9).

$$
\begin{gathered}
B=C_{P} \frac{\left(T_{S}-T_{\infty}\right)}{h_{g f}} \\
S=\frac{h}{C_{P} \cdot G} \\
X=L / r_{0} \\
Y=\left(\dot{m} / m_{0}\right)^{1 / 2}
\end{gathered}
$$

At this point, namely Equation (5), [1] comes to a crossroad, since $G$ and $S$ depend on $Y$, and for this, Equation (5) cannot be integrated. A pure analytic result is not achievable. However, assuming $G_{M}$ and $S_{M}$ as constant, this author proposed the correlation, Equation (10):

$$
X=\left(\frac{G_{0}}{G_{M}}\right)^{1 / 2} \cdot S_{M}^{-1} \cdot B^{-1}
$$

And $S_{M}$ is described by Equation (11):

$$
S_{M}=\frac{h}{C_{P} \cdot G_{M}}
$$

Considering the results of their experiment, treated by linear regression, the numeric format of Equation (12) was proposed, within 13.6\% accuracy.

$G_{M}$ was arbitrarily chosen as $275 \mathrm{~kg} / \mathrm{m}^{2} / \mathrm{s}$, constant value, related to the critical vapor mass rate of the nozzle, as a representative value of the order of the magnitude of the mass rate. The value of 1.932 for $S_{M}$ in Equation (12), is partially originated from the arbitrarily chosen value of $275 \mathrm{~kg} / \mathrm{m} / \mathrm{s}^{2}$ and the conditions of the experiment ( $h$, and $C_{P}$ ), which Kerney [1] derived in their experiment, considering a linear fit of the achieved data.

$$
X=\frac{1}{1.932}\left(\frac{G_{0}}{G_{M}}\right)^{1 / 2} \cdot B^{-1}
$$

In [3], a method to extend the semi-empirical correlation scope, in order to present results considering fluids other than water, and pressures other than ambient in the pool was presented. To achieve it, the proposed correlation considers the influence of the fluid density. 
The development is not analogous to that in [3] since it uses a full set of equation (continuity of mass, linear momentum, and energy) while [1] used only the continuity of mass. Another theoretical remark is that the former considered that there is a cross flow of vapor to the bath, which is condensed within it, while the latter considered the opposite entrainment of water into the vapor jet, which is partially evaporated, and creates a two-phase flow zone.

This development also presented a point where the integration of the continuity of the mass is not possible since the integrand is not a defined function of the mass cross flow. An approximated correlation is considered, and this further development, considering experimental data, yields:

$$
X=35.5\left(\frac{G_{0}}{G_{W}}\right)^{1 / 2} \cdot B^{-1} \cdot\left(\frac{\rho_{\infty}}{\rho_{w}}\right)^{-1 / 2}
$$

where $G_{W}$ is not an arbitrary fixed value since it was considered the mass flux in the point where the jet finishes an isentropic expansion. After that, the jet allows entrained water in. The " $W$ " properties are calculated considering the laws of the Thermodynamics, for the depth in which the jet starts the two-phase flow. The average absolute deviation found was $21.9 \%$, higher than the value found in the precedent work.

A slightly better average absolute deviation is found both by Kerney [1] and Weimer [3], when they depart of the condition to have a priori fixed value exponents, and consider them a free product of the regression. No further development of the latter was found in the literature.

Based on previous work, Chun [4], consider that the characteristics of the jet are mainly dependent on 1) the degree of subcooling of the bath, 2) the steam mass flux, 3) the nozzle direction and 4) the depth of the nozzle. The efficiency of the Direct Contact Condensation as a mechanism of heat transfer is also praised, although attention is called to the fact that no reliable correlation to determine the length of the jet exists. According to this paper, much of the disagreement is related to the fact that, experimentally, the length of the jet is obtained by a visual method, what raises issues related to the geometric limits of the jet. In this paper, the end of the jet was considered as the interface between pure vapor and two-phase flow regions. Theoretical development was not presented, and this paper focused on the development of new values for old parameters. The theoretical expression has its roots in Kerney [1], as shown in Equation (14).

$$
X=F\left(\frac{G_{0}}{G_{M}}, B, S_{M}\right)
$$

Empirically, through visual method, the values of $X$ are determined, which allows Chun [4] propose Equation (15), within a $20 \%$ dispersion,

$$
X=0.5923 \cdot\left(\frac{G_{0}}{G_{M}}\right)^{0.3444} \cdot B^{-0.66}
$$


In sequence, as product of a regression, it is also proposed a correlation for the heat transfer coefficient, Equation (16):

$$
\frac{h}{C_{P} \cdot G_{M}}=0.8012 \cdot\left(\frac{G_{0}}{G_{M}}\right)^{0.3444} \cdot X^{-1.0079} B^{-0.6247}
$$

The value of $G_{M}$ is also not mentioned throughout the work, for what is supposed that in [4], Chun considered the fixed value of $275 \mathrm{~kg} / \mathrm{m}^{2} / \mathrm{s}$ as formerly proposed.

Proceeding a similar experiment (vapor injection in a subcooled water pool, atmospheric pool), reference Kim, et al. (2001) produced the correlation as presented in Equation (17) and Equation (18):

$$
\begin{gathered}
X=0.503 \cdot\left(\frac{G_{0}}{G_{M}}\right)^{0.47688} \cdot B^{-0.70127} \\
\frac{h}{C_{P} \cdot G_{M}}=1.4453 \cdot\left(\frac{G_{0}}{G_{M}}\right)^{0.13315} \cdot B^{0.03587}
\end{gathered}
$$

The value of $G_{M}$ was again considered $275 \mathrm{~kg} / \mathrm{m}^{2} / \mathrm{s}$, as originally proposed. It is worth to notice that Equation (10), Equation (15) and Equation (17) are reasonably similar in their forms. Both are directly obtained from the original mathematical development.

Gulawani, in [5] and Kang, in [6] present CFD as a new tool to perform the geometric analysis of the jet, and its heat transfer coefficient. Shah, in [7], also by CFD analysis, found heat transfer coefficients ranging between 0,6 and 08 $\mathrm{MW} / \mathrm{m}^{2} / \mathrm{K}$ and a dimensionless length ranging between 3.8 and 8 .

In [8], it was experimentally proposed a different form of correlation. For instance, this model includes a pressure correction factor, as shown in Equation (21):

$$
X=0.868\left(\frac{P_{0}}{P_{a}}\right)^{0.2} \cdot\left(\frac{G_{0}}{G_{M}}\right)^{0.5} \cdot B^{-0.60}
$$

This correlation is within a $40 \%$ band of error.

This development leads to the proposal of another heat transfer correlation, as shown in Equation (22):

$$
S_{M}=\frac{h}{C_{P} \cdot G_{M}}=0.576\left(\frac{P_{0}}{P_{a}}\right)^{0.2} \cdot\left(\frac{G_{0}}{G_{M}}\right)^{0.5} \cdot B^{-0.4}
$$

$\mathrm{Xu}$, in [9] proposed a different configuration of an experiment when a vertical pipe injected vapor in a flow of water instead of a pool. The extension and the heat transfer coefficient correlations were determined to have the Reynolds number as a parameter. The results presented the dimensionless extension between 0.29 and 4.64 and the heat transfer coefficient between $0.34 \mathrm{MW} / \mathrm{m}^{2} \mathrm{~K}$ and $11.36 \mathrm{MW} / \mathrm{m}^{2} \mathrm{~K}$.

Chong, in [10] proposed a correlation for a straight-pipe nozzle, based on the original formulation. Under this work, the dimensionless extension correlation, 
the geometry of the orifice nozzle was considered, through the imposition of a geometrical factor $\left(\varepsilon / \varepsilon^{\prime}\right)^{2}$, which represents the expansion ratio between a straight-line nozzle and actual orifice nozzle (for straight-line nozzle, $\left(\varepsilon / \varepsilon^{\prime}\right)^{2}=1$ ), as presented in Equation (21).

$$
X=0.3866\left(\frac{\varepsilon}{\epsilon}\right)^{2} \cdot\left(\frac{G_{0}}{G_{M}}\right)^{0.78} \cdot B^{-0.80}
$$

As a summary, Table 1 presents the main correlations for determining the dimensionless length in the literature, within the scope of this work.

One can notice that the constant value of $G_{M}=275 \mathrm{~kg} / \mathrm{s}$ is broadly found (up to 2015) in the literature ([1] [4] [5] [7] [8] [9] [11] and [10]). For each one of these, $S_{M}$ takes a different constant value, valid only for the respective correlation and for the respective range of data. A more realistic formulation of $G_{M}$ would be of great interest since it directly impacts the formulation of $X, S_{M}$, and $h$.

\section{Development}

The experimental works under this scope are related to the development of a correlation for the non-dimensional length and heat transfer coefficient, through the propositions of variations of the originally proposed development from which results in the length correlation (Equation (10)). The extension of the works studied shows concern related to increasing the accuracy in a variety of experimental settings and parameters, in order to reduce the band of adjustment numerical error, since no fully analytical model is yet available.

The band of the error band is determined by fluctuations, which were neglected when Equation (5) was approximated. Sonin, in [12] experimentally investigated this phenomenon related to pressure waves propagating along the pool, while Youn [13] focused the particular case in which pressure waves are

Table 1. Extension correlation selected in the literature.

\begin{tabular}{rl}
\hline Dimensionless Extension & Equation Number \\
$X=0.5200\left(\frac{G_{0}}{G_{M}}\right)^{1 / 2} \cdot B^{-1}$ & $35.5\left(\frac{G_{0}}{G_{w}}\right)^{1 / 2} \cdot B^{-1} \cdot\left(\frac{\rho_{\infty}}{\rho_{w}}\right)^{-1 / 2}$ \\
$X$ & $=0.5923 \cdot\left(\frac{G_{0}}{G_{M}}\right)^{0.3444} \cdot B^{-0.66}$ \\
$X$ & $=0.503 \cdot\left(\frac{G_{0}}{G_{M}}\right)^{0.4768878} \cdot B^{-0.70127}$ \\
$X$ & $=0.868\left(\frac{P_{S}}{P_{a}}\right)^{0.2} \cdot\left(\frac{G_{0}}{G_{M}}\right)^{0.5} \cdot B^{-0.60}$ \\
$X$ & $=0.3866\left(\frac{\varepsilon}{\epsilon}\right)^{2} \cdot\left(\frac{G_{0}}{G_{M}}\right)^{0.78} \cdot B^{-0.80}$
\end{tabular}


created by a low mass flux of injection. The case where multiple jets are simultaneously created by different holes was explored by Cho [14]. The fluctuations related to turbulence, for example, were not originally considered in the analytical development of the semi-empirical correlations. This analysis indicates that some fluctuation is intrinsic to the process and offers an open field to research.

The experimental works follow a standard: the length of the jet is observed, what allows the definition of a correlation of length and the determination of the heat transfer coefficient. The experimental data collected supplies complementary information in order to complement the development of Equation (5).

In order to achieve a more realistic value of $G_{M}$, this paper proposes a new correlation. This value is developed through the application of the $1^{\text {st }}$ and $2^{\text {nd }}$ law of the thermodynamics in the jet, considering it an isentropic discharge, and a function of the conditions in the pressure chamber, as displayed from Equation (24) to Equation (30):

$$
\begin{gathered}
G_{M}=\rho_{x} \cdot V_{\text {Crit }} \\
V_{\text {Crit }}=\sqrt{2 \cdot\left(h_{0}-h_{\text {crit }}\right)} \\
h_{0}=h\left(P_{0}, T_{0}\right) \\
h_{\text {crit }}=h\left(P_{\text {crit }}, S_{\text {crit }}\right) \\
P_{\text {crit }}=0.577 \cdot P_{0} \\
\rho_{x}=\rho\left(101.3 \mathrm{kPa}, S_{\text {Crit }}\right) \\
S_{\text {crit }}=s_{0}
\end{gathered}
$$

Within the scope of this paper, the proposed correlations of $G_{M}$ (Equation (24)) substituted the formerly constant value of $G_{M}=275 \mathrm{~kg} / \mathrm{m}^{2} / \mathrm{s}$ in those correlations presented in Table 1 . The numerical analysis that follows considered the experimental data obtained from Kerney [1].

\section{Results}

\section{1) The Validity of $G_{M}$ as Product of an Isentropic Process}

Table 2 presents some important data related to the numerical procedure. Item (i) presents the least square adjustment error when this procedure is applied to the correlation in the respective literature. All the correlations in item (i) were applied to the experimental data presented in Kerney [1]. Item (ii) presents the adjustment error found when the least square procedure is applied to the correspondent correlation when using the value of $G_{M}$ as proposed in Equation (24), as suggested by this paper. The experimental data comes also from Kerney [1]. The percentage difference between (i) and (ii) is presented in item (iii). Item (iv) presents the value of $S_{M}$ as found in the respective literature, while (v) presents $S_{M}$ recalculated, where the data originally used by each respective author is swapped by the data presented in Kerney [1]. The difference from (iv) and (v) can be explained as a consequence of the fact that each correlation stands only for the range of data of the experiment for what it was developed. So, 
changing the parameters of the experiment (e.g. the temperature of the pool, pressure of injection, mass flux, etc.), the correlation would also have to be changed in its parameters $\left(S_{M}\right.$, and the exponents of $\left.G_{0} / G_{M}, B\right)$. This is evidenced with the small difference for the column of Equation (12), but greater for the remaining columns. Last item, (vi), evaluates the value of $S_{M}$ as a product of the isentropic relation in Equation (24). The difference between correspondent values of references (v) and (vi) is small, indicating that the isentropic correlation stands for a broad range of parameters.

For the analysis of Table 2, it is evidenced by the dependence of $S_{M}$ on the range of parameters employed in the experiment, since items (iv) and (v) present a large difference. When the isentropic formulation of $G_{M}$ (Equation (24)) is used in item (vi), this difference is vastly reduced (see items (v) and (vi)).

The currently proposed distribution of $G_{M}$ as an isentropic relation (Equation (24)), applied to the experimental data within Kerney [1], follows a logarithmic path along the pressure in the chamber axis $\left(P_{C}\right)$, as presented in Figure 3. Equation (30) presents $G_{M}=G_{M}\left(P_{C}\right)$ as a product of regression.

$$
G_{M}=127.6367+26.4510 \cdot \ln \left(P_{C}\right)
$$

The graphics in Figures 4-7 present comparisons between the correlations proposed by the respective authors, considering $G_{M}=275 \mathrm{~kg} / \mathrm{m}^{2} / \mathrm{s}$ constant, as originally proposed by Kerney [1] versus the currently proposed correlation for $G_{M}$, within the scope of this paper, Equation (24). The respective results were plotted against the experimental data.

As noticed, the correlations considering both propositions of $G_{M}$ found a narrow agreement, what validates the use of $G_{M}$ as the product of an isentropic assumption (Equation 24) as proposed by the present paper.

\section{2) Heat Transfer Coefficient}

Once the value of $G_{M}$ is specified by Equation (24), the heat transfer coefficient $(h)$ can be analytically derived from Equation (11), in conjunction with the adjusted value of $S_{M}$, which is obtained from the linear coefficient of the least square adjustment in the correlations of Table 1. In this section, this value of heat transfer coefficient, this way obtained, is compared with values found

Table 2. Numerical parameters of the method.

\begin{tabular}{|c|c|c|c|c|c|}
\hline Item & Description & $\begin{array}{l}\text { Equation } \\
\text { (12) }\end{array}$ & $\begin{array}{l}\text { Equation } \\
\text { (15) }\end{array}$ & $\begin{array}{l}\text { Equation } \\
\quad(17)\end{array}$ & $\begin{array}{l}\text { Equation } \\
\quad(21)\end{array}$ \\
\hline (i) & Adjustment Error $\left(G_{M}=275 \mathrm{~kg} / \mathrm{m}^{2} / \mathrm{s}\right)$ & 2.268 & 2.762 & 2.241 & 2.949 \\
\hline (ii) & Adjustment Error ( $G_{M}$ as in Equation 24) & 2.409 & 2.907 & 2.407 & 2.458 \\
\hline (iii) & Difference in Percentage (i) and (ii) & $6.255 \%$ & $5.273 \%$ & $7.403 \%$ & $16.65 \%$ \\
\hline (iv) & $\begin{array}{c}S_{M} \text { literature, calculate by the respective author, and } \\
\text { with data from the respective author }\end{array}$ & 1.923077 & 1.688334 & 1.988072 & 1.152074 \\
\hline (v) & $S_{M}\left(G_{M}=275 \mathrm{~kg} / \mathrm{m}^{2} / \mathrm{s}\right)$ recalculated with Kerney [1] & 1.918281 & 0.59453 & 0.776398 & 0.534474 \\
\hline (vi) & $\begin{array}{l}S_{M}\left(G_{M} \text { as in Equation } 24\right), \text { calculate with data from } \\
\text { Kerney [1] }\end{array}$ & 1.850481 & 0.581395 & 0.749625 & 0.514933 \\
\hline
\end{tabular}




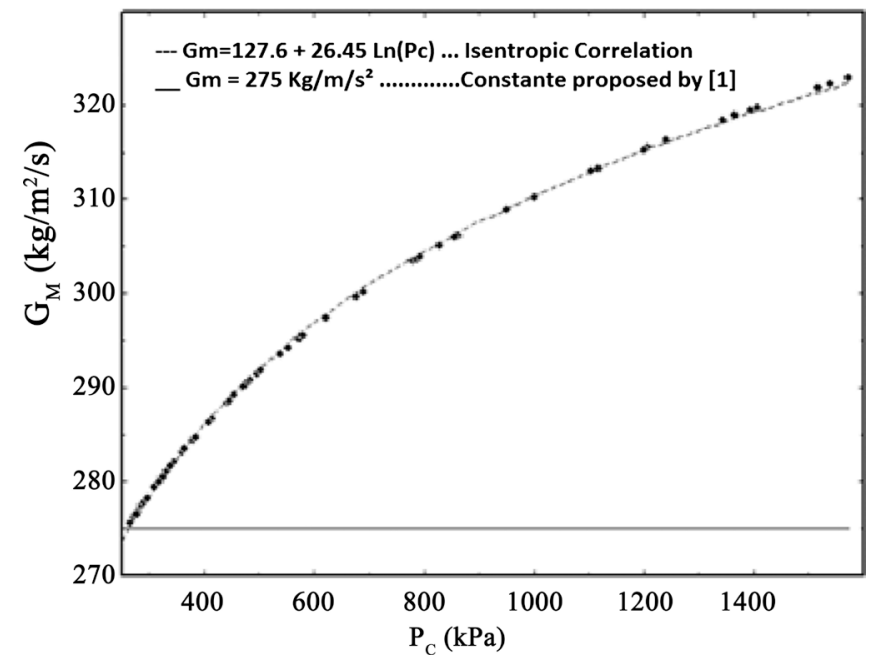

Figure 3. $G_{M}$ as isentropic relation (Equation (24)) vs. pressure in the chamber $\left(P_{C}\right)$ for Kerney [1], experimental data.

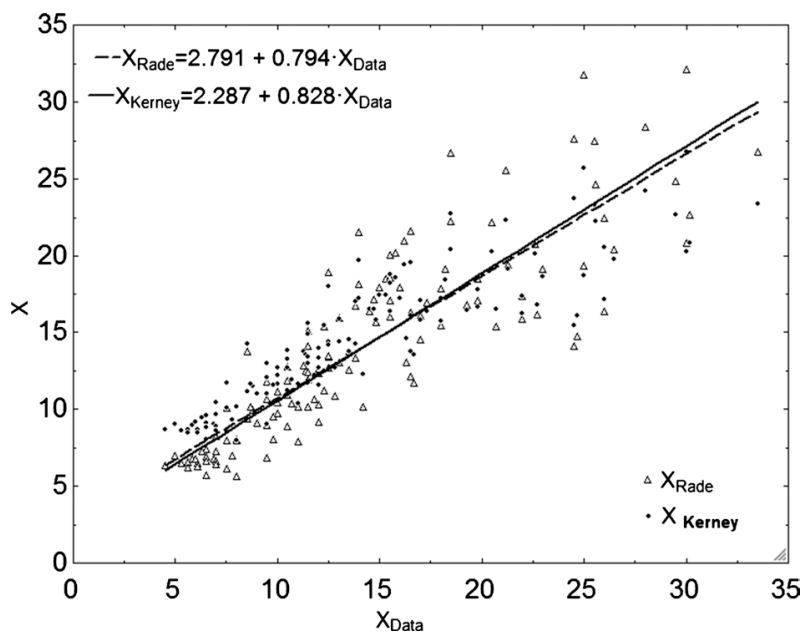

Figure 4. Correlation of Kerney [1], vs. Data $X$ dispersion using $G_{M}=275 \mathrm{~kg} / \mathrm{m}^{2} / \mathrm{s}$ constant and by $G_{M}$ as an isentropic formulation (Equation (24)).

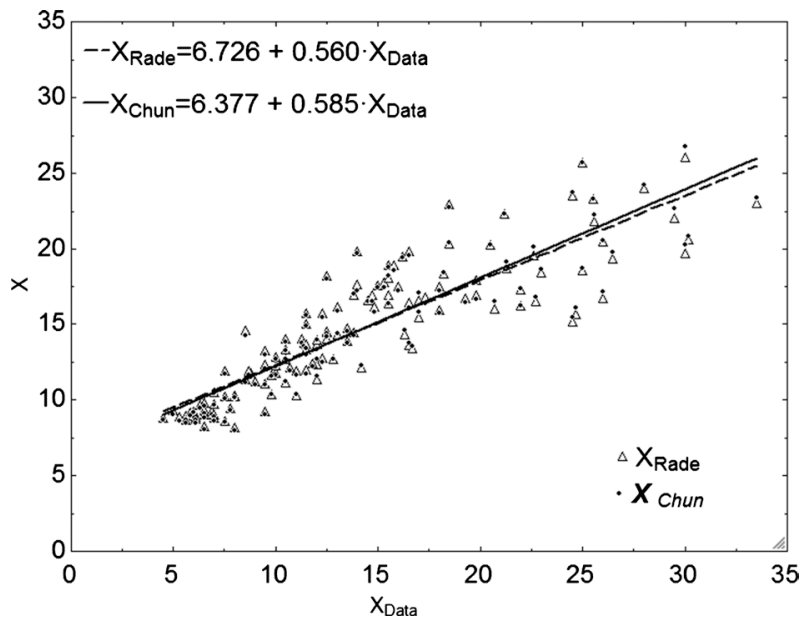

Figure 5. Correlation of Chun [4], vs. Data ( $X$ dispersion using $G_{M}=275 \mathrm{~kg} / \mathrm{m}^{2} / \mathrm{s}$ constant and $G_{M}$ as an isentropic formulation, Equation (24)). 


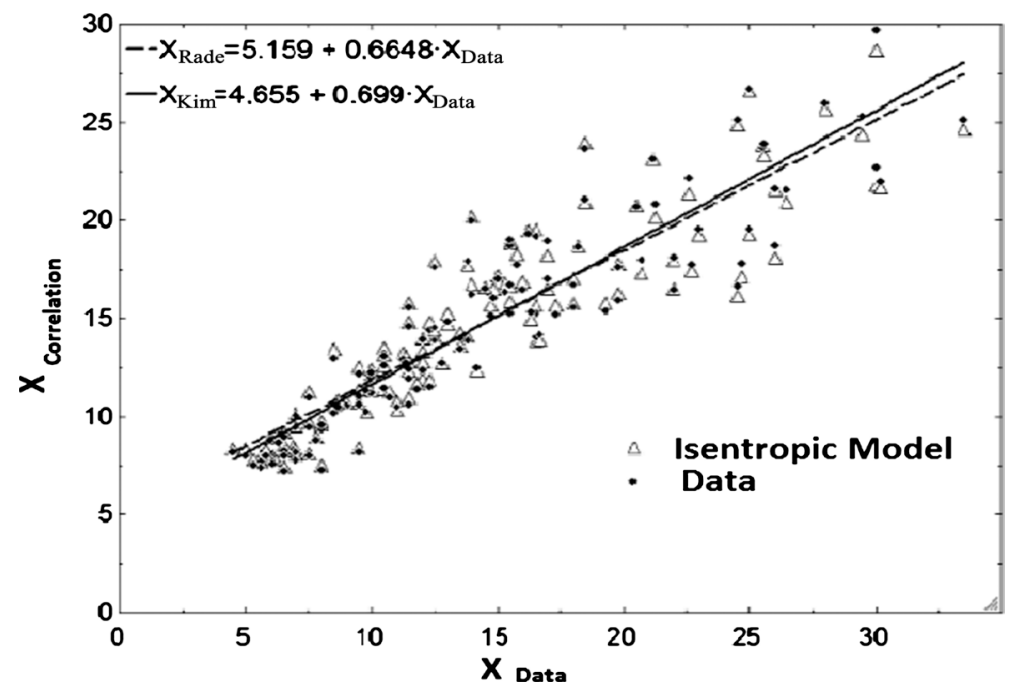

Figure 6. Correlation Kim [11], vs. Data ( $X$ dispersion using $G_{M}=275 \mathrm{~kg} / \mathrm{m}^{2} / \mathrm{s}$ constant and $G_{M}$ as an isentropic formulation, Equation (24)).

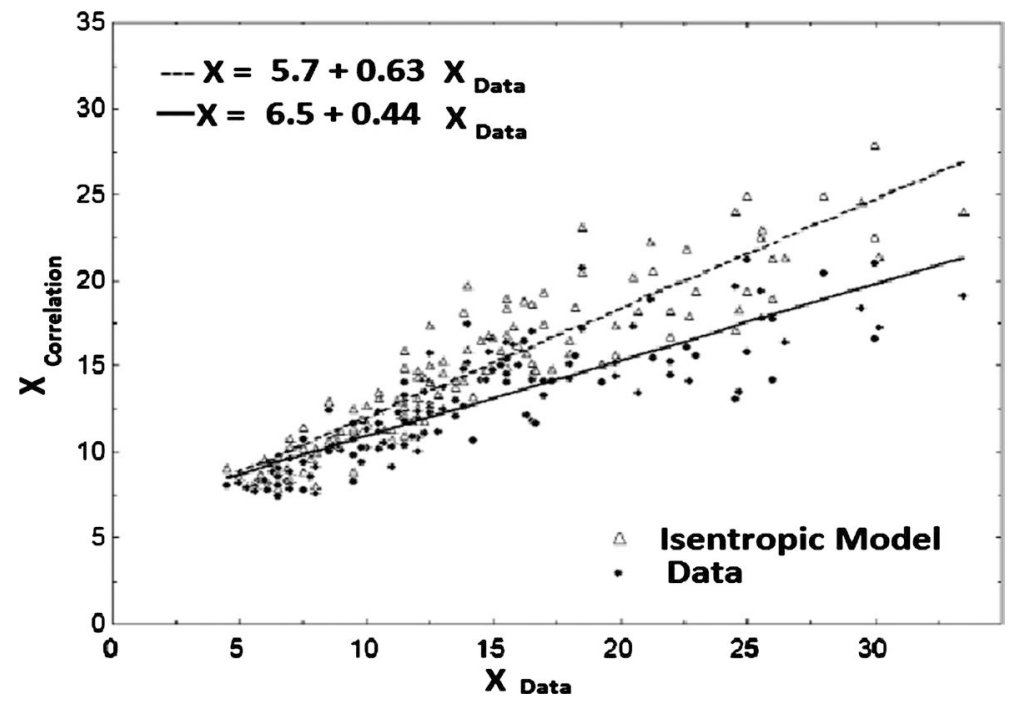

Figure 7. Correlation of Wu [8], vs. Data ( $X$ dispersion using $G_{M}=275 \mathrm{~kg} / \mathrm{m}^{2} / \mathrm{s}$ constant and $G_{M}$ as an isentropic formulation, Equation (24)).

through the application of correlations in the literature (Equation (16) and Equation (18)).

Figure 8 and Figure 9 present $h$ as a smooth function of $P_{C}$. Both sets of data belong to the same magnitude order, which indicates that this procedure to obtain $h$ may be valid.

In Figure 9, difference decreases as long as the pressure in the chamber increases. On the other hand, Figure 10 and Figure 11 present the value of the heat transfer coefficient, found through the application of the least square procedure in an extension correlation in order to determine $M$ with data from Kerney [1]. These values are plotted vs $G_{0}$. When " $h$ " is represented as a function of " $G_{0}$ ", its smoothly dependence on " $G_{0}$ " is easily observed. Once more, both results belong to the same magnitude order. 


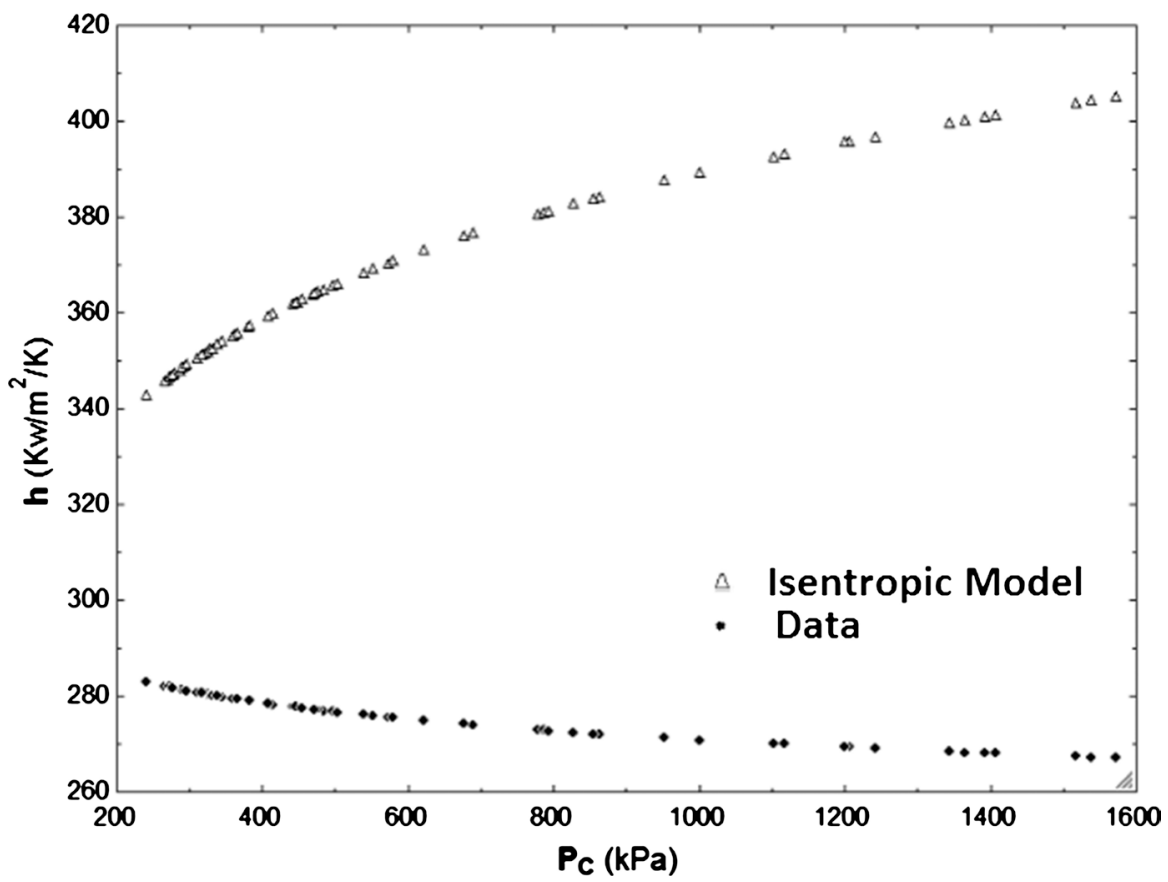

Figure 8. Heat Transfer Coefficient vs Pressure in the Chamber. $G_{M}$ from an isentropic relation (Equation (24)); $h$ from the correlation of Chun [4], Equation (16); Data from Kerney [1].

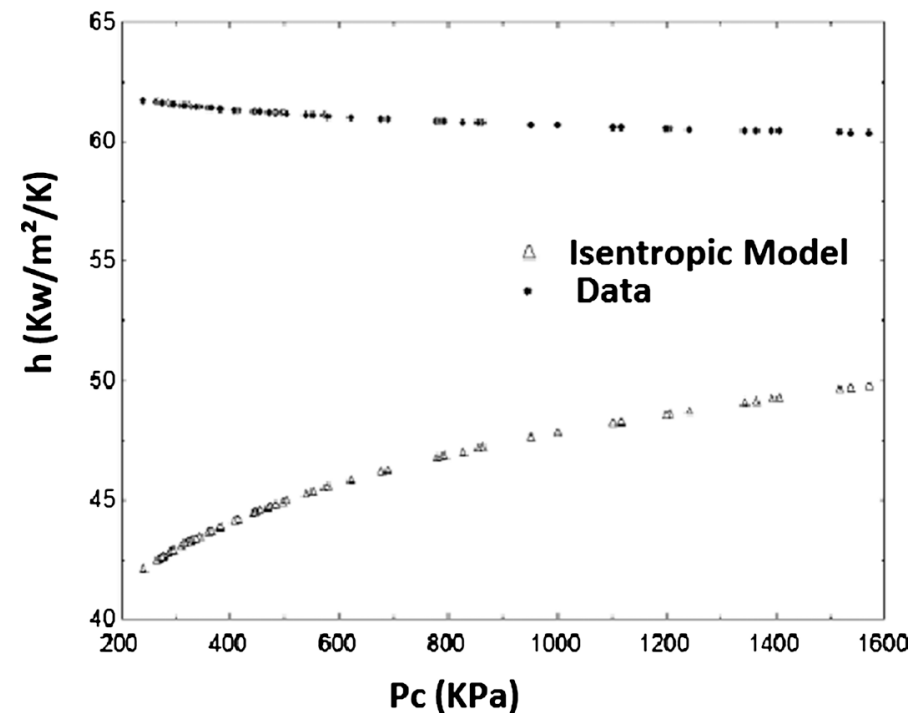

Figure 9. Heat Transfer Coefficient vs Pressure in the Chamber. $G_{M}$ from an isentropic relation (Equation (24)); $h$ from the correlation of Kim [11], Equation (18); Data from Kerney [1].

\section{Conclusions}

The decision to maintain $S_{M}$ as a constant is numerically satisfactory since the chosen value is adjusted by the linear regression to fit the found data. Through this premise, any value proposed for $G_{M}$ would generate the same adjustment error. This decision, proposed originally by Kerney [1], has been assumed up to works in 2015, although it does not depict the physics of the problem. 


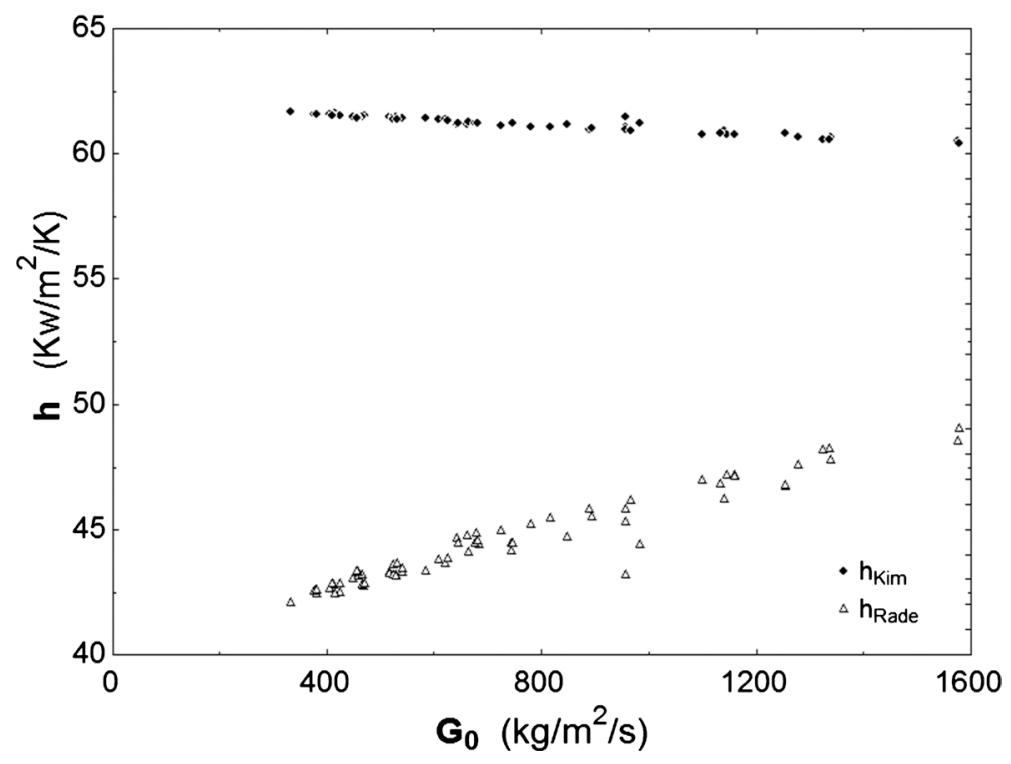

Figure 10. Heat Transfer Coefficient considering $G_{M}$ from Equation (24) vs. mass flux ratio. $h$ from correlation of Chun [4] vs. mass flux ratio. Data from Kerney [1].

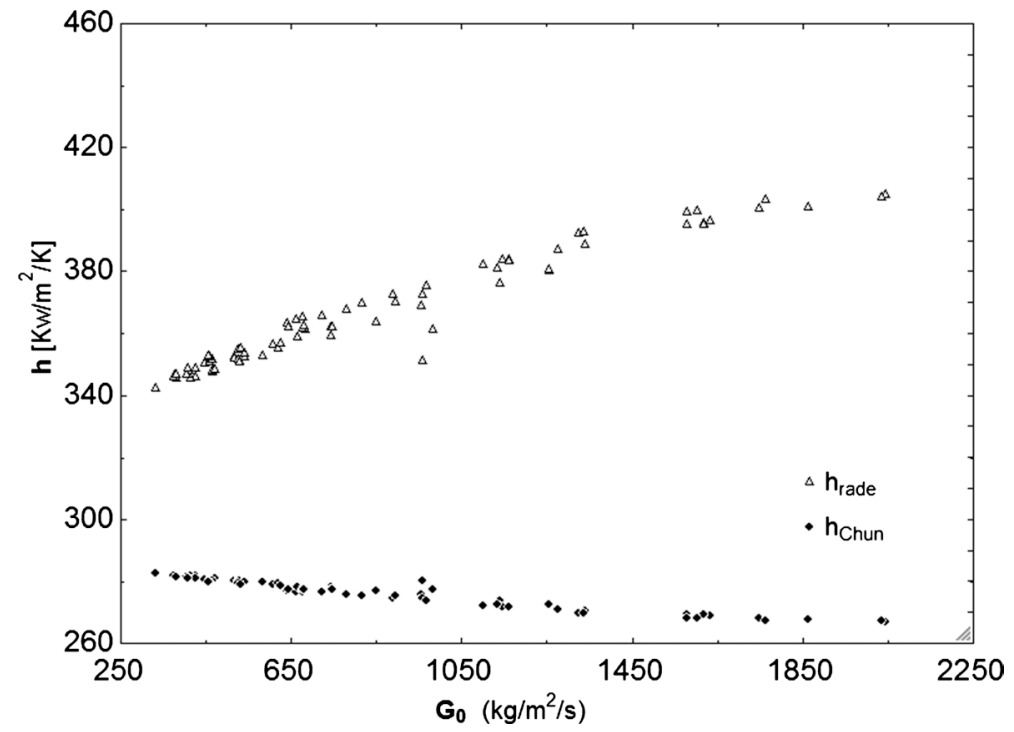

Figure 11. Heat Transfer Coefficient considering $G_{M}$ from Equation (24) vs. mass flux ratio. $h$ from the correlation of Kim [11] vs. mass flux ratio. Data from Kerney [1].

A full analytic function is not possible to be achieved since the area and the variation of major parameters as a function of the flux of mass in Equation (5) are unknown. These gaps have been fulfilled with experimental data, allowing the propositions of correlations, which present some degree of adjustment error, and are suitable only for the range of the experimented data.

This work presented satisfactory arguments to question the original formulation, where the mass flow rate $\left(G_{M}\right)$ is constant. This original formulation stands as reasonable when a formulation for the extension of the jet is proposed since any constant value proposed fits when applied the linear regression. Although it presents the following drawbacks: 1 ) the formulation is only valid for the specific 
range of data for what it was created; 2) it does not allow the analytical evaluation of the heat transfer coefficient from the extension equation, Equation (10).

This way, the isentropic formulation of $G_{M}$ adds flexibility for the extension jet equation, once it is less dependent of experimental data, and allows the analytical evaluation of the heat transfer coefficient from the extension equation.

Considering the scope of correlations presented in this work, the proposed correlation of $G_{M}$ as an isentropic function of the pressure chamber stands as reasonable and would apply to any extension of thermodynamical conditions.

Besides, the isentropic formulation of $G_{M}$ allows the direct deduction of the heat transfer coefficient from the formulation of the extension, which reduces the dependence on experimental data. The present analysis indicates the possibility to reduce the dependence on experimental data to determine $h$, and points to a direction where more experimental efforts could be expended.

Further works could focus on the reinforcement of the presented correlation through the experimental analysis, considering a large range of parameters.

\section{Acknowledgements}

The authors would like to acknowledge Nuclear and Energy Research Institute, IPEN-CNEN/SP and CTMSP/SP for the infrastructure, particularly the computer laboratory.

\section{Conflicts of Interest}

The authors declare no conflicts of interest regarding the publication of this paper.

\section{References}

[1] Kerney, P.J., Faeth, G.M. and Olson, D.R. (1972) Penetration Characteristics of a Submerged Steam Jet. AIChE Journal, 18, 548-553.

https://doi.org/10.1002/aic.690180314

[2] van Wissen, R.J.E., Schreel, K.R.A.M., van der Geld, C.W.M. and Wieringa, J. (2004) Turbulence Production by a Steam-Driven Jet in a Water Vessel. International Journal of Heat and Fluid Flow, 25, 173-179. https://doi.org/10.1016/j.ijheatfluidflow.2003.11.013

[3] Weimer, J.C., Faeth, G.M. and Olson, D.R. (1973) Penetration of Vapor Jets Submerged in Subcooled Liquids. AIChE Journal, 19, 552-558. https://doi.org/10.1002/aic.690190321

[4] Chun, M.-H., Kim, Y.-S. and Park, J.-W. (1996) An Investigation of Direct Condensation of the Steam Jet in Subcooled Water. International Communications in Heat and Mass Transfer, 23, 947-958. https://doi.org/10.1016/0735-1933(96)00077-2

[5] Gulawani, S.S., Joshi, J.B., Shah, M.S., RamaPrasad, C.S. and Shukla, D.S. (2006) CFD Analysis of Flow Pattern and Heat Transfer in Direct Contact Steam Condensation. Chemical Engineering Science, 61, 5204-5220. https://doi.org/10.1016/j.ces.2006.03.032

[6] Kang, H.-S. and Song, C.-H. (2008) CFD Analysis for Thermal Mixing in a Sub- 
cooled Water Tank under a High Steam Mass Flux Discharge Condition. Nuclear Engineering and Design, 238, 492-501. https://doi.org/10.1016/j.nucengdes.2007.02.044

[7] Shah, A., Chughtai, I.R. and Ham, M. (2010) Numerical Simulation of Direct-Contact Condensation from a Supersonic Steam Jet in Subcooled Water. Chinese Journal of Chemical Engineering, 18, 577-587. https://doi.org/10.1016/S1004-9541(10)60261-3

[8] Wu, X.-Z., Yan, J.-J., Shao, S.-F., Cao, Y. and Liu, J.-P. (2007) Experimental Study on the Condensation of Supersonic Steam Jet Submerged in Quiescent Subcooled Water: Steam Plume Shape and Heat Transfer. International Journal of Multiphase Flow, 33, 1296-1307. https://doi.org/10.1016/j.ijmultiphaseflow.2007.06.004

[9] Xu, Q., Guo, L., Zou, S., Chen, J. and Zhang, X. (2013) Experimental Study on Direct Contact Condensation of a Stable Steam Jet in Water Flow in a Vertical Pipe. International Journal of Heat and Mass Transfer, 66, 808-817.

https://doi.org/10.1016/j.ijheatmasstransfer.2013.07.083

[10] Chong, D.-T., Zhao, Q.-B., Yuan, F., Wang, W., Chen, W. and Yan, J.-J. (2015) Research on the Steam Jet Length with Different Nozzle Structures. Experimental Thermal and Fluid Science, 64, 134-141. https://doi.org/10.1016/j.expthermflusci.2015.02.015

[11] Kim, H.-Y., Bae, Y.-Y., Song, C.-H. and Park, J.-K. (2001) Experimental Study on Stable Steam Condensation in a Quenching Tank. International Journal of Energy Research, 25, 239-252. https://doi.org/10.1002/er.675

[12] Sonin, A.-A. (1981) Scaling Laws for Small-Scale Modeling of Steam Relief into Water Pools. Nuclear Engineering and Design, 65, 17-21. https://doi.org/10.1016/0029-5493(81)90115-1

[13] Youn, D.-H., Ko, K.-B., Lee, Y.-Y., Kim, M.-H., Bae Y.-Y. and Park, J.-K. (2012) The Direct Contact Condensation of Steam in a Pool at Low Mass Flux. Journal of Nuclear Science and Technology, 40, 881-885. https://doi.org/10.1080/18811248.2003.9715431

[14] Cho, S., Chun, S.-Y., Baek, W.-P. and Kim, Y. (2004) Effect of Multiple Holes on the performance of Sparger during Direct Contact Condensation of Steam. Experimental Thermal and Fluid Science, 28, 629-638. https://doi.org/10.1016/j.expthermflusci.2003.10.002 


\section{Nomenclature}

$A$ Heat Transfer Area $\left(\mathrm{m}^{2}\right)$

$B$ Condensation Driving Potential

$C_{p}$ Water Specific Heat $\left(\mathrm{J} / \mathrm{kg} /{ }^{\circ} \mathrm{C}\right)$

$G$ Steam Mass Flux $\left(\mathrm{kg} / \mathrm{m}^{2} / \mathrm{s}\right)$

$h$ Average Heat Transfer Coefficient $\left(\mathrm{W} / \mathrm{m}^{2 \circ} \mathrm{C}\right)$

$h_{f g}$ Condensation Enthalpy $(\mathrm{kJ} / \mathrm{kg})$

$L \quad$ Steam Jet Length (m)

$\dot{m}$ Vapor Flow Rate $(\mathrm{kg} / \mathrm{s})$

$P \quad$ Pressure $(\mathrm{kPa})$

$r \quad$ Jet Radius (m)

$R$ Rate of Condensation $\left(\mathrm{kg} / \mathrm{m} / \mathrm{s}^{2}\right)$

$S$ Dimensionless Transport Modulus

$T$ Temperature $\left({ }^{\circ} \mathrm{C}\right)$

$x \quad$ Axial Coordinate $(\mathrm{m})$

$X$ Non-Dimensional Jet Length

$\rho$ Density $\left(\mathrm{kg} / \mathrm{m}^{3}\right)$

\section{Subscripts}

$A$ Atmospheric Conditions

$C$ Conditions in the Pressure Chamber

$f$ Conditions of Saturated Liquid

$S$ Conditions in the Vapor-Bath Interface

$g$ Conditions of Saturated Vapor

$\infty$ Average Conditions in the Pool, Far Away from the Jet

0 Conditions in the Nozzle

$M$ Average Conditions over the Interface Surface

$W$ Developed as [3] 